\title{
PRINCIPLES AND TREATMENT APPROACHES OF DYSLIPIDAEMIAS: A REVIEW
}

\section{Datta Debranjan*}

\author{
Department of Pharmacology, Sikkim Manipal Institute of Medical Sciences, Tadong, Gangtok, Sikkim,
}

*Corresponding Author's E.mail: debranjan_datta@live.com

\begin{abstract}
Dyslipidaemia is a common clinical problem globally. It includes abnormality in LDL cholesterol, HDL cholesterol and triglyceride levels. The prevalence of dyslipidaemia observed higher in males than females among urban Indian adults. ${ }^{1}$ Hypertension and dyslipidaemia are well established risk factor for various cardiovascular diseases. There is frequent coexistence of hypertension and hypercholesterolemia. ${ }^{2}$ This prevalence varies globally. Causes of dyslipidaemia are either primary disorders of lipoproteins or secondary to metabolic causes. In addition to metabolic syndrome, other causes of combined dyslipidaemia are lack of physical activity, hypothyroidism, diabetes mellitus etc. Clinicalconditions such as metabolic syndromes, dyslipidaemia, hypertension, early atherosclerosis and type 2 diabetes results due to Overweight and obesity in childhood and adolescents. Familial hypercholesterolemia is predominant during childhood with elevated Low Density Lipoprotein cholesterol (LDL-C). Hypertriglyceridemia are elevations of either chylomicron, intermediate density lipoproteins (IDL) or very low density lipoproteins (VLDL). Combined dyslipidaemia include abnormalities of severe lipoproteins, usually elevated LDL-C and triglyceride (TG) with low high density lipoprotein cholesterol (LDL-C). Non pharmacologic management of several dyslipidaemias include lifestyle modification, regular exercise, weight management and nutritional approach. Pharmacologic management with statin is individualised based on overall risk, pattern of dyslipidaemia and associated medical conditions. Combination medication to treat cholesterol may be effective when a single agent is ineffective or there is a need to reduce the dosage of medication.
\end{abstract}

Key Words: Dyslipidaemia, familial hypercholesterolemia, lipoproteins hypertension, statins.

\section{INTRODUCTION}

Cardiovascular diseases (CVD) are the major cause of death globally. Dyslipidaemia is one of the major risk factor for the CVD, along with other factors such as hypertension, smoking, obesity etc. Dyslipidaemias are primary disorders of lipoprotein metabolism (due to genetic reason) or secondary disorders induced by other metabolic causes such as diabetes mellitus, hypothyroidism or hypopituitarism. Dyslipidaemias are a common clinical problem which includes either lipoprotein overproduction or its deficiencies. Mixed dyslipidaemia is characterised by elevated levels of triglycerides (TG) and reduced High Density Lipoprotein cholesterols (HDL-C), with or without elevated levels of low density lipoprotein cholesterol (LDL-C). These metabolic abnormalities contribute to increased risk for CVD. Lipoproteins are spherical particles of water soluble proteins, which transport TGs, cholesterols $(\mathrm{CH})$ and cholesterol esters (CE) through body fluids. A metabolic disorder of lipoproteins causing elevation in any kind of lipoproteins is called hyperlipidaemias or hyperlipoproteinemias. Itis a major cause of acute pancreatitis and atherosclerosis. ${ }^{3}$ The first is more prevalent in patients with hyperlipaemia, which is characterised sby very high level of TG. The term 'dyslipidaemia' is nowadays preferred over hyperlipidaemia or hyperlipoproteinemia. Lipoproteins act synergistically with various environmental and metabolic factors to promote atherosclerosis and thrombosis. They are smoking, diabetes mellitus, obesity, and high blood pressure. Anunderlying relationship between serum lipoprotein concentration and atherosclerosis is evidenced by various epidemiologic, pathologic, animal, genetic and metabolic studies. ${ }^{4}$ Hyperlipidaemia is also major cause of atherosclerosis-associated conditions such as coronary heart disease, ischemic cerebrovascular disease, and peripheral vascular disease. Atherosclerosis and atherosclerosis -associated conditions are leading cause of (C) 2011, JDDT. All Rights Reserved death globally and in particular western countries. Lipoproteins that contain apo-lipoprotein B 100 such as LDL, VLDL, lipoprotein-a transport lipids into the artery wall, causing slow occlusion of coronary vessels and thereby leading to formation of thrombi. On the other hand, HDLretrieve cholesterol from the artery wall and inhibit oxidation of atherogenic lipoproteins. Thereby HDL exerts antiatherogenic effect. Therefore it can be stated that low level of HDL (in case of cigarette smoking) and oxidative stress (in case if diabetes mellitus)are risk factor for atherosclerotic diseases. Measurement of plasma apo-lipoprotein B 100 concentration and non HDL-C become important parameter for understanding dyslipidaemia. Effective treatment of dyslipidaemias either by pharmacological or non-pharmacological interventions has shown reduced cardiovascular disease morbidity and mortality in many trials. ${ }^{5-7}$ Although LDL-C is the main culprit for the lipoprotein associated risk, but LDL-C level does not reflect the classic diabetic dyslipidaemias. HIV infection as well as its treatment is linked with abnormalities in lipid metabolism. Patients with HIV associated fat redistribution are more likely to have dyslipidaemia, possibly related to excess visceral adipose tissue accumulation. ${ }^{8}$

\section{DYSLIPIDAEMIA CLASSIFICATION}

Dyslipidaemia is classified into four categories (as listed in table below) based on clinical, genetic and biochemical parameters. Each of these four categories has a specific therapeutic approach.

1. Elevated LDL-C: Elevation of LDL-C, without an elevation of TG is considered as primary or familial hypercholesterolemia (FH). It occurs due to defects in LDL receptor gene. This affects approximately 1 in 500 Americans, and 1 in 200 French Canadians. 
2. Very high TG: Fasting TG level over $1000 \mathrm{mg} / \mathrm{dl}$ can be due to genetic influences, or a combination of genetic predisposition and secondary causes such as alcohol abuse, poorly controlled diabetes, obesity, renal disease etc. ${ }^{9}$ They have relatively low risk of CHD, but have a high risk of pancreatitis, mostly due to lipemia and subsequent hyper viscosity. ${ }^{10}$

3. Low HDL-C: HDL-cholesterol is a most prevailing predictor of premature CHD along with thrombotic risk factors among young Indians and Americans. ${ }^{11,12}$ Low HDL-C is associated with genetic factors, male sex, hypertriglyceridemia, smoking, obesity. Familial hypoalphalipoproteinemia is prevalent in approximately 10 per cent of CHD patients aged below 60 years. Within families affected by early and apparently genetic CHD, dyslipidaemias have a much higher occurrence than that in the general population. A $20 \%$ $30 \%$ of early familial CHD has been attributed to primary hypoalphalipoproteinemia (low HDL-C). ${ }^{13}$

4. Combined dyslipidaemias: It is most prevalent in patients who survive myocardial infarction or suffering from coronary revascularization. It includes abnormalities of several lipoproteins, mainly elevated LDL-C and TG with low HDL-C. This disorder does not become apparent until adulthood.

Table 1: Classification of dyslipidaemias

\begin{tabular}{lll}
\hline Lipoprotein levels (mg/dl) & Fredrickson-Levy class & Genetic disorder \\
\hline LDL-C elevated & II A & $\begin{array}{l}\text { Familial hypercholesterolemia (LDL>200) } \\
\text { Primary hypercholesterolemia (LDL 130-199) }\end{array}$ \\
LDL-C $>130$ & & \\
TG ${ }^{* *} 150$ & & \\
HDL-C $>40$ & I, V & LPL deficiency \\
TG elevated & & Apo C-III deficiency \\
TG $>500$ & & Familial hypertriglyceridemia \\
LDL-C NA ${ }^{* * * *}$ & & \\
HDL-C NA & - & Hypo- $\alpha$-lipoproteinemia \\
HDL-C decreased & & \\
HDL-C $<40$ & & \\
TG $<150$ & II B, IV, III & \\
LDL-C $<130$ & & Familial combined hyperlipidemia \\
Combined dyslipidaemias & & Familial dysbetalipoproteinemia \\
LDL-C $>130$ & & \\
TG $>150$ & & \\
HDL-C $<40$ & & \\
\hline
\end{tabular}

${ }^{*} L D L-C=L o w$ density lipoprotein- Cholesterol, $* * T G=$ Triglycerides, $* * H D L-C=H i g h$ density lipoprotein-Cholesterol, $* * * * N A=N o t$ applicable.

\section{SCREENING GUIDELINES FOR DETECTION OF DYSLIPIDAEMIAS}

As per American Association of Clinical Endocrinologists (AACE) Clinical Practice Guideline, screening for detection of dyslipidaemia can be done through several stages. First and foremost steps are identifying the risk factors and categorize them. This will maximize the treatment effectiveness. Major risk factors include advancing age, high serum total cholesterol level, high non HDL -C level, established coronary artery diseases (CAD), family history of CAD, presence of hypertension, diabetes mellitus, cigarette smoking. Additional risk factors include obesity, elevated apo B, polycystic ovary syndrome in women etc. It is important to determine the 10 year risk of a coronary event using various tools such as Framingham Risk assessment Tool or by Reynolds Risk score. ${ }^{(14)}$ AACEClinical Practice Guideline recommends that special attention to be given for assessing women for CAD risk. AACE screening recommendations are based on age and risk, not based on sex, therefore, women should be screened in the same way as men. As per AACE recommendation, early diagnosis and management of paediatric dyslipidaemia to reduce the levels of LDL-C that may eventually increase risk of cardiovascular events in adulthood need to be carried out. AACE also recommends more frequent assessments for all patients with a family history of premature CAD (myocardial infarction or sudden death before 55 years of age in father or other male first-degree relative, or before 65 years of age in mother or other female first-degree relative). Adult patients with diabetes mellitus should be screened annually for dyslipidaemia. All adults of 20 years of age should be evaluated every 5 years as part of a global risk assessment for dyslipidaemia. In the absence of CAD risk factors, middle-aged persons (Men Aged 45-65 Years, Women Aged 55-65 Years) should be screened for dyslipidaemia at least every 1 to 2 years. Adults older than 65 years of age should undergo lipid assessment if they have multiple CAD risk factors. AACE also recommends that children older than 2 years of age should be screened every 3 to 5 years if they have CAD risk factors or having a family history of premature CAD or dyslipidaemia, or have insulin resistance syndrome, or have no available family history.

\section{SCREENING TESTS RECOMMENDATION}

Various screening tests are recommended by AACE for the detection of cardiovascular risks. ${ }^{(14)}$

1. Fasting lipid profile: This includes total cholesterol, LDL-C, triglycerides, and HDL-C.

2. Low-Density Lipoprotein Cholesterol measurement: AACE recommends direct measurement of LDL-C in certain high-risk patients, such as those with 
fasting TG levels greater than $250 \mathrm{mg} / \mathrm{dl}$ or those with diabetes mellitus or known vascular disease.

3. High-Density Lipoprotein Cholesterol measurement: AACE recommends this test as a screening test for dyslipidaemia. An HDL-C concentration greater than $60 \mathrm{mg} / \mathrm{dl}$ is an independent negative risk factor in both sexes.

4. Non High-Density Lipoprotein Cholesterol measurement: If insulin resistance is suspected, AACE recommends evaluation of non-HDL-C to know about patient's total atherogenic lipoprotein burden. In addition, when TGs are $200 \mathrm{mg} / \mathrm{dl}$ or greater but less than 500 $\mathrm{mg} / \mathrm{dl}$, a non HDL-C calculation will provide better risk assessment than LDL-C alone.

5. Triglyceride estimation: AACE recommends screening of TGs as a component of lipid screening as elevated TG level is an important risk factor for CAD.

6. Apolipoprotein estimation: AACE recommends Apo B measurements to assess the success of LDL-C lowering therapy.

\section{AACE TREATMENT RECOMMENDATIONS}

Goal is to attempt for lipid levels in the range of normal; however, more aggressive goals can be set for higher-risk patients. AACE recommends a broad strategy to control lipid levels and associated metabolic abnormalities and also to limit risk factors such as hypertension, diabetes, obesity, and cigarette smoking. Most cost effective treatment approach to dyslipidaemias and CAD is nonpharmacological interventions. Although pharmacotherapy is recommended for those patients who are at highest risk and fail to reach desiredcholesterol level after an adequate trial of lifestyle changes in 6 months.

\section{Pharmacotherapy:}

Currently available lipid lowering drugs include HMGCoA reductase inhibitors (statins), fibric acid derivatives (fibrates), nicotinic acid (niacin), bile acid sequestrates, and cholesterol absorption inhibitors (ezetimibe). For isolated LDL-C elevations or elevated LDL-C after TG is controlled with combined dyslipidaemias, statins are the first drug of choice, although bile acid resins, psyllium have proven efficacy with gastrointestinal side effects. A number of intervention trials have shown that among statins, atorvastatin is most cost-effective for moderate to high risk patients. ${ }^{15}$ After treating secondary causes for TG $>400-500 \mathrm{mg} / \mathrm{dl}$, niacin and gemfibrozil is the drug of choice. Fibrates are the choice of drug for hypertriglyceridemia associated with diabetes, gout etc. While TGs are being reduced with gemfibrozil, there is associated increase in LDL-C level. This can be treated with another fibrate group of drug fenofibrate. Omega 3 fish oil capsule also lower TGs effectively, although can cause abdominal bloating, flatulence, diarrhoea. Mixed dyslipidaemia is frequently associated with glucose intolerance for which niacin is not effective. Otherwise statin, combining fibrate and psyllium are effective treatment approach. On the other hand isolated low HDL$\mathrm{C}$ are slow to response to treatment and for which only low to moderate dose niacin is the drug of choice. Patients on medications should be monitored every 4 to 6 weeks to adjust dose and evaluate side effects until lipid goals are achieved.

\section{Nonpharmacological interventions:}

This includes nutrition and regular exercise, weight management, and cessation of smoking.

\section{Exercise:}

Exercise remains an essential approach for dyslipidaemia control and cardiovascular risk factor reduction, as evidenced by various published guidelines. Physical activity is associated with improvement in lipoprotein profile. Daily physical activity increases the clearance of TG-rich lipoproteins and glucose. Exercise of moderate intensity, longer duration and increased frequency appears to have more benefit for dyslipidaemia and weight loss than higher intensity, less frequent activity. AACE recommends at least 30 minutes of moderate intensity physical activity (consuming $4-7 \mathrm{kcal} / \mathrm{min}$ ) 4 to 6 times weekly, with an expenditure of at least $200 \mathrm{kcal} / \mathrm{day}$. Physical activities include brisk walking, aerobics, cleaning/scrubbing; mowing the lawn and various sporting activities such as basketball, or volleyball with light effort. Recent guidelines indicate that greater benefits are achieved when the duration of exercise is lengthened to 60 to 90 minutes daily. ${ }^{16}$ ross-sectional evidence indicates that there is association between dyslipidaemia and reduced metabolic clearance of insulin (MCRI). ${ }^{17}$ Regular physical activity such as weight and resistance training also improves insulin sensitivity.

\section{Nutrition and weight management:}

Proper nutrition therapy provides an important instrument for the management of dyslipidaemia. The U.S. dietary guidelines and the food pyramid recommend choice of food that will reduce the risk of CVD in general population, and form basis for guidelines to treat dyslipidaemias. ${ }^{18}$ Dietary fat comprises both saturated as well as unsaturated fatty acids. The substitution of unsaturated fatty acids with saturated fatty acids leads to decreased LDL-C levels. High intake of poly unsaturated fatty acids reducesthe HDL-C and triglyceride levels. Substitution of monounsaturated fatty acids for saturated fatty acids has a minimal effect on HDL-C values and does not raise triglyceride levels. Whereas dietary intake of trans-fatty acids is associated with both increased LDL-C and decreased HDL-C levels. Current nutritional guidelines for the reduction of cardiovascular risk through lipid management recommend diets rich in fruits, vegetables, grains, legumes, high fibre cereals, low-fat dairy products, fish, lean meats, and skinless poultry. Guidelines also indicate that poly unsaturated and monounsaturated fatty acids may comprise up to $10 \%$ and $20 \%$ of caloric intake, respectively, and that total dietary fat should constitute $25 \%$ to $35 \%$ of calories consumed. Diet recommendation for dyslipidaemia should be individualised based on patient's lipid pattern, body weight, food preference, level of motivation. Patients with hypercholesterolemia are less responsive to diet changes than those with other forms of hypercholesterolemia. Including 20 to $30 \mathrm{~g}$ of dietary fibre per day, of which 10 to $25 \mathrm{~g}$ is soluble, could result in an additional $5 \%$ to $15 \%$ reduction in TChol and LDL-C. Patients with combined dyslipidaemia and metabolic syndrome should control their weight in an proper manner and do regular exercise, as these changes will have a favourable impact on all serum lipoproteins, glucose intolerance and abnormal obesity. A $10 \%$ reduction in body weight is associated 
Datta et al

Journal of Drug Delivery \& Therapeutics; 2013, 3(2), 141-144

with up to a $30 \%$ reduction in abnormal obesity. A patient should achieve this by limiting total fat intake to $25 \%$ to $30 \%$ of calories and saturated fat to less than $7 \%$ of calories. Total carbohydrate intake should not exceed $60 \%$ of calories.

\section{Smoking cessation:}

\section{REFERENCES}

1. Sawant AM, Shetty D, Mankeshwar R AT. Prevalence of dyslipidemia in young adult Indian population. $\mathrm{J}$ Assoc Physicians India. 56:99-102.

2. Williams RR, Hunt SC, Hopkins PN, Stults BM, Wu LL, Hasstedt SJ et al. Familial dyslipidemic hypertension: Evidence from 58 Utah families for a syndrome present in approximately $12 \%$ of patients with essential hypertension. JAMA. 1988;259:3579-86.

3. Katzang, Masters T. Basic and clinical Pharmacology. 11th ed. Tata Mc Graw-Hill; 2009. p. 605.

4. LaRosa JC, Hunninghake D, Bush D et al. The cholesterol facts. A summary of the evidence relating dietary fats, serum cholesterol, and coronary heart disease. A joint statement by the American Heart Association and the National Heart, Lung, and Blood Institute. The task force on cholesterol Iss. 81st ed. American Heart Association. 1990. p. 1721-33.

5. Nishiwaki M, Ikewaki K, Ayaori M, Mizuno K, Ohashi Y, Ohsuzu F IT, H N. Risk reductions for cardiovascular disease with pravastatin treatment by dyslipidemia phenotype: A post hoc analysis of the MEGA Study. J Cardiol. 2012;(Dec).

6. Millán J, Alegría E, Guijarro C, Lozano JV, Vitale GC G-TB, JR G-J. Dyslipemia in diabetics treated with statins. Results of the Dyslipidemia International Study in Spain. Med Clin (Barc). 2012;(Dec).

7. Scandinavian Simvastatin Survival Study Group. Randamised trial of cholesterol lowering in 4444 patients with coronary heart disease. Lancet. 1994;344(9):1383.

8. Hadigan C, Meigs J, Corcoran C et al. HIV/AIDSMAJOR ARTICLE: metabolic abnormalities and cardiovascular disease risk factors in adults with human immunodeficiency virus infection and lipodystrophy. Clin Infect Dis. 2001;32:130-9.

9. RH K. Drug treatment of lipid disorder. New England Journal of Medicine. 1999;341:498-511. 14. 2011;134(July):26-32. Obes Metab.
Cigarette smoking is associated with a number of metabolic processes that affect lipoproteins, including increasing plasma free fatty acids, glucose and VLDL-C, and lowering HDL-C. Smoking cessation is associated with an average increase in HDL-C of 6 to $8 \mathrm{mg} / \mathrm{dl} .{ }^{19}$

10. Viljoen A WA. Diagnosis and treatment of severe hypertriglyceridemia. Expert Rev Cardiovasc Ther. 10(4):505-

11. Panwar RB, Gupta R, Gupta BK, Raja S, Vaishnav J, Khatri M AA. Atherothrombotic risk factors \& premature coronary heart disease in India: a case-control study. Indian J Med Res.

12. Boden WE. High density lipoprotein cholesterol as an independent risk facor in cardiovascular disease: assessing the data from Framingham to the Veterans affairs High density lipoprotein Intervention Trial. Am J Cardiol. 2000;86:19L-22L.

13. E N Kort, D G Ballinger, W Ding, S C Hunt, B R Bowen, V Abkevich, K Bulka, B Campbell, C Capener, A Gutin, K Harshman, M McDermott, T Thorne, H Wang, B Wardell, J Wong, P N Hopkins, M Skolnick and MS. Evidence of linkage of familial hypoalphalipoproteinemia to a novel locus on chromosome 11q23. Am J Hum Genet. 66(6):1845-56.

14. Jellinger PS, Smith DA. AACE Lipid and Atherosclerosis Guidelines. Endocrine Practice. 2012;18(Supplyment 1).

15. Plosker GL L-WK. Atorvastatin: a pharmacoeconomic review of its use in the primary and secondary prevention of cardiovascular events. Pharmacoeconomics. 2007;25:1031-53.

16. Krauss RM, Eckel RH, Howard B et al. AHA Dietary Guidelines: revision 2000: A statement for healthcare professionals from the Nutrition Committee of the American Heart Association. Circulation. 2000;102(2284-2299).

17. Christine Lee C, Lorenzo C, Haffner SM, Wagenknecht LE, Goodarzi MO S, D, Norris JM, Rewers MJ HA. Components of metabolic syndrome and 5-year change in insulin clearance The Insulin Resistance Atherosclerosis Study (IRAS). Diabetes

18. DHHS. Dietary guidelines for Americans. 5th ed. USDA and US department of health and Human services.; 2000.

19. McBride PE. The health consequences of smoking. Cardiovascular diseases. Med Clin North Am. 1992;76:333-53. 